VoL. 41 (1990) [393-405]

\title{
ON CONSTRAINED STOCHASTIC OPTIMAL PARAMETER SELECTION PROBLEMS
}

\author{
C.J. GoH AND K.L. TEO
}

\begin{abstract}
This paper considers a special class of stochastic optimal parameter selection problem subject to probability constraints on the state. The system dynamics are governed by a linear Ito stochastic differential equation with controllable parameters appearing nonlinearly in the dynamics. The problem seeks to optimise a cost functional which is quadratic in the state with weighting matrices being time invariant but depending nonlinearly on the parameters. Although the inclusion of the probability state constraints renders the problem insolvable by the conventional LQG theory, we show that the problem can in fact be transformed into an equivalent deterministic optimal parameter selection problem solvable by an existing software MISER. Numerical examples are presented to demonstrate the feasibility and efficiency of the proposed approach.
\end{abstract}

\section{INTRODUCTION}

The importance of optimal parameter selection problems has attracted attention both from the practical engineering application as well as the mathematical interest point of view. Necessary conditions for optimality for both deterministic and stochastic systems have been derived in $[2,16]$ while computational methods are furnished in $[6,13,18]$. It should be noted that very few results on optimal parameter selection problems are allowed to include explicit constraints on the state vector. Two exceptions are found in [6] and [18].

In $[13,16]$, a class of stochastic optimal parameter selection problems is transformed into a class of deterministic problems involving linear parabolic partial differential equations, where the state variables of the stochastic problems correspond naturally to the spatial variables of their deterministic counterparts. Nevertheless, due to the inherent difficulty in solving a PDE numerically, there are difficulties in using this approach to solve problems with more than two state variables.

This paper considers a dynamic system governed by a linear Ito stochastic differential equation with nonlinear dependence on some decision parameters. These parameters are selected so as to minimise the expected value of the cost functional defined by the definite integral of some quadratic function of the state vector. The aim of the

Received 9 June 1989

Copyright Clearance Centre, Inc. Serial-fee code: 0004-9729/90 \$A2.00+0.00. 
paper is two-fold. Firstly we show that, by a transformation similar to that of [14], the stochastic problem can be reduced to a deterministic problem involving only ordinary differential equations. This is in contrast to the PDE approach of $[13,16]$ and hence the curse of dimensionality can be alleviated somewhat. Secondly, the peculiar feature of probability constraints on the state vector is also shown to be reducible to a deterministic continuous state constraint.

The form of these constraints is similar to the cost functional considered in [19] though the solution method proposed therein is again via the PDE approach of $[13$, 16]. The overall probabilistically constrained problem can thus be reduced to a form easily amenable to an existing software MISER $[8,9]$.

\section{ThEORETICAL FORMULATION}

Consider a system described by the following linear Ito stochastic differential equation defined on the fixed time interval $(0, T]$ :

$$
d \xi(t)=A(K) \xi(t) d t+D(K) d w(t)
$$

with the prescribed initial condition

$$
\xi(0)=\xi^{0},
$$

where $\xi(t)=\left[\xi_{1}(t), \ldots, \xi_{n}(t)\right]^{T} \in \mathrm{R}^{n}$ is the state vector, $K=\left[K_{1}, \ldots, K_{N}\right]^{T} \in \mathbb{R}^{N}$ is the parameter vector, and $\xi^{0}=\left[\xi_{1}^{0}, \ldots, \xi_{n}^{0}\right]^{T} \in \mathrm{R}^{n}$ is the initial state vector which is Gaussian distributed with mean $\mu^{0}$ and covariance $M^{0} \in \mathrm{R}^{n \times n}$. Furthermore, $A(K) \in$ $\mathrm{R}^{n \times n}$ and $D(K) \in \mathrm{R}^{n \times m}$ are continuously differentiable matrix-valued functions of the parameter vector $K$; and $w(t)=\left[w_{1}(t), \ldots, w_{n}(t)\right]^{T} \in \mathbf{R}^{m}$ is a Wiener process with zero mean and covariance

$$
E\left\{w(t) w^{T}(\tau)\right\}=\int_{0}^{\min (t, \tau)} \Theta(s) d s,
$$

where $\Theta \in \mathbf{R}^{m \times m}$ is a symmetric positive definite matrix function. We assume that the Wiener process $w(t)$ and the initial random vector $\xi^{0}$ are statistically independent.

Define

$$
\Xi \equiv\left\{K \in \mathbf{R}^{N}: h_{j}(K) \leqslant 0, \quad j=1, \ldots, r\right\}
$$

where $h_{j}, j=1, \ldots, r$, are continuously differentiable functions of the parameter $K$.

To continue, let us introduce an important class of constraints on the state of the stochastic dynamical system (1). A special class of these constraints requires the state 
to be in a given acceptable region with prescribed degree of confidence for all $t \in[0, T]$. The general class of these probabilistic state constraints may be stated as follows:

$$
\text { Prob }\left\{a_{i} \leqslant\left(C^{i}\right)^{T} \xi(t) \leqslant b_{i}, t \in[0, T]\right\} \geqslant \triangle_{i}, i=1, \ldots, p
$$

where $C^{i}, i=1, \ldots, p$, are $n$-vectors, $a_{i}, i=1, \ldots, p, b_{i}, i=1, \ldots, p$, and $\triangle_{i}$, $i=1, \ldots, p$, are real constants.

An element from $\Xi$ is said to be a feasible parameter if it satisfies the probabilistic state constraints specified in (4). Let $\mathcal{F}$ be the class of all such feasible parameters.

The stochastic optimal parameter selection problem is formally posed as follows:

Subject to the dynamical system (1), find a feasible parameter vector $K \in \mathcal{F}$ such that the following cost functional:

$$
J(K)=E\left\{\xi^{T}(T) S(K) \xi(T)+\int_{0}^{T} \xi^{T}(t) Q(K) \xi(t) d t\right\}
$$

is minimised over $\mathcal{F}$, where $S(K) \in \mathbf{R}^{n \times n}$ and $Q(K) \in \mathbf{R}^{n \times n}$ are symmetric, positive semi-definite matrices continuously differentiable with respect to $K$. For convenience, let this optimal parameter selection problem be referred to as the problem $(P)$.

Note that solution to the problem $(P)$ without the probabilistic state constraints and/or parameter constraints has been well established in the literature $[14,3,11,12]$ in the context of linear quadratic state feedback control. However, it appears that no computational algorithm is available for solving the problem $(P)$ in the present general setting.

\section{Deterministic transformation}

In this section, we show, by an approach similar to that of [14], that the optimal parameter selection problem (P) is equivalent to a deterministic one. To begin, we note that for each $K$, the solution of the system (1) can be written as

$$
\xi(t \mid K)=\Phi(t, 0 \mid K) \xi^{0}(K)+\int_{0}^{t} \Phi(t, s \mid K) D(K) d w(s)
$$

where, corresponding to each $K, \Phi(t, \tau \mid K)$ is the principal solution matrix of the homogeneous system:

$$
\begin{aligned}
\frac{\partial \Phi(t, \tau)}{\partial t} & =A(K) \Phi(t, \tau) \\
\Phi(\tau, \tau) & =I
\end{aligned}
$$

where $I$ is the identity matrix. 
It is well-known that for each $K$ and for each $t \in[0, \infty), \xi(t \mid K)$ is a Gaussian random vector. Its mean is given by

$$
\mu(t \mid K)=E(\xi(t \mid K))=\Phi(t, 0 \mid K) \mu^{0}
$$

while its covariance matrix is given by

$$
\begin{aligned}
\psi(t \mid K)= & E\left\{(\xi(t \mid K)-\mu(t \mid K))(\xi(t \mid K)-\mu(t \mid K))^{T}\right\} \\
= & \Phi(t, 0 \mid K) M^{0}(\Phi(t, 0 \mid K))^{T} \\
& +\int_{0}^{t} \Psi(t, \tau \mid K) D(K) \Theta(\tau)(D(K))^{T}(\Psi(t, \tau \mid K))^{T} d \tau
\end{aligned}
$$

It follows from (7), (8) and (9) that $\mu(t \mid K)$ and $\psi(t \mid K)$ satisfy the following differential equations:

$$
\begin{aligned}
\frac{d \mu(t)}{d t} & =A(K) \mu(t), \\
\mu(0) & =\mu^{0}
\end{aligned}
$$

and

$$
\begin{aligned}
\frac{d \psi(t)}{d t} & =A(K) \psi(t)+\psi(t)(A(t))^{T}+D(K) \Theta(t)(D(K))^{T} \\
\psi(0) & =M^{0}
\end{aligned}
$$

Note that there are only $n(n+1) / 2$ independent differential equations in (11) by virtue of its symmetry.

The corresponding conditional joint probability density function for $\xi(t)$ is given by

$$
\begin{aligned}
& f\left(x, t \mid \xi^{0}, K\right) \\
& =(2 \pi)^{-n / 2}[\operatorname{det} \psi(t \mid K)]^{-1 / 2} \exp \left\{-\frac{1}{2}(x-\mu(t \mid K))^{T} \psi^{-1}(t \mid K)(x-\mu(t \mid K))\right\}
\end{aligned}
$$

Let us now turn our attention to the cost functional (5). First we note that

$$
\begin{aligned}
E\left[\xi^{T} Q \xi\right] & =E\left[\operatorname{Tr}\left(\xi^{T} Q \xi\right)\right]=E\left[\operatorname{Tr}\left(Q \xi \xi^{T}\right)\right]=\operatorname{Tr}\left[Q E\left(\xi \xi^{T}\right)\right] \\
& =\operatorname{Tr}\left[Q(K)\left(\psi(t \mid K)+\mu(t \mid K) \mu(t \mid K)^{T}\right)\right]
\end{aligned}
$$

The first term of the cost functional (5) can be converted in a similar manner. Consequently, we have 
LEMMA 1. The cost functional (5) is equivalent to

$$
\begin{aligned}
J(K)= & \operatorname{Tr}\left\{S(K)\left[\psi(T \mid K)+\mu(T \mid K)(\mu(T \mid K))^{T}\right]\right\} \\
& +\int_{0}^{T} \operatorname{Tr}\left\{Q(K)\left[\psi(t \mid K)+\mu(t \mid K)(\mu(t \mid K))^{T}\right]\right\} d t
\end{aligned}
$$

where $\mu(t \mid K)$ and $\psi(t \mid K)$ are deterministic and are determined, respectively, by (10) and (11).

For the probabilistic state constraints specified in (4), we have

Lemma 2. For each $i=1, \ldots, p$, the corresponding probabilistic constraint specified in (4) is equivalent to

$$
\operatorname{erf}\left\{\frac{b_{i}-\left(C^{i}\right)^{T} \mu(t \mid K)}{\left(2\left(C^{i}\right)^{T} \psi(t \mid K) C^{i}\right)^{1 / 2}}\right\}-\operatorname{erf}\left\{\frac{a_{i}-\left(C^{i}\right)^{T} \mu(t \mid K)}{\left(2\left(C^{i}\right)^{T} \psi(t \mid K) C^{i}\right)^{1 / 2}}\right\} \geqslant 2 \triangle_{i}
$$

for all $t \in[0, T]$.

Proof: Since $\xi(t)$ is Gaussian with mean $\mu(t \mid K)$ and covariance $\psi(t \mid K)$, it is clear that for each $i=1, \ldots, p$, the scalar process $\left(C^{i}\right)^{T} \xi(t)$ is also Gaussian but with mean $\left(C^{i}\right)^{T} \mu(t \mid K)$ and variance $\left(C^{i}\right)^{T} \psi(t \mid K) C^{i}$. Thus, the corresponding constraint specified in (4) can be rewritten as

$$
\int_{a_{i}}^{b_{i}} \frac{1}{\left(2 \pi\left(C^{i}\right)^{T} \psi(t \mid K) C^{i}\right)^{1 / 2}} \exp \left\{-\frac{1}{2} \frac{\left(y-\left(C^{i}\right)^{T} \mu(t \mid K)\right)^{2}}{\left(C^{i}\right)^{T} \psi(t \mid K) C^{i}}\right\} d y \geqslant \triangle_{i}
$$

The constraint (15) is thus obtained readily by direct integration of (16).

Note that each of the constraints specified in (15) is required to be satisfied for all $t \in[0, T]$; this constitutes an infinite dimensional constraint.

Invoking the constraint transcription introduced in $[8,17]$, the constraint $(15)$ is equivalent to

$$
\begin{aligned}
G_{i}(K)= & \eta \int_{0}^{T}\left(\operatorname { M i n } \left\{\operatorname{erf}\left[\frac{b_{i}-\left(C^{i}\right)^{T} \mu(t \mid K)}{\left(2\left(C^{i}\right)^{T} \psi(t \mid K) C^{i}\right)^{1 / 2}}\right]\right.\right. \\
& \left.\left.-\operatorname{erf}\left[\frac{a_{i}-\left(C^{i}\right)^{T} \mu(t \mid K)}{\left(2\left(C^{i}\right)^{T} \psi(t \mid K) C^{i}\right)^{1 / 2}}\right]-2 \triangle_{i}, 0\right\}\right)^{2} d t=0,
\end{aligned}
$$


where $\eta$ is an empirical constant introduced to improve the accuracy of satisfaction of the constraints concerned. Note also that each of these constraints is in the same form as the cost functional $(5)$. In $[8,18]$ this is referred to as being in the canonical form. It is necessary to remark here that this constraint transcription, although computationally feasible, suffers from the disadvantage of not being able to satisfy the usual constraint qualification condition [17]. An improved version of this transcription which overcomes this inadequacy is furnished in $[\mathbf{1 0}]$.

Let $\phi(t)$ be the vector formed from $\mu(t)$ and the independent components of the matrix $\psi(t \mid K)$, and let $f$ be the corresponding vector obtained from the right-handside of (10) and (11). Furthermore, let $\mathcal{F}$ again denote the class of all feasible parameters in the sense that each of its elements is in $\Xi$ and satisfies the constraints specified in (17). The above results can be summarised in the following theorem.

THEOREM 1. The problem $(P)$ is equivalent to the following standard deterministic optimal parameter selection problem:

Subject to the dynamical system:

$$
\begin{aligned}
\frac{d \phi(t)}{d t} & =f(\phi(t), K, t) \\
\phi(0) & =\phi^{0},
\end{aligned}
$$

where $\phi^{0}$ is formed by $\mu^{0}$ and the components appearing in the upper triangular part of the covariance matrix $M^{0}$, find a parameter vector $K \in \mathcal{F}$ such that the cost functional:

$$
J(K)=\Gamma(\phi(T \mid K), K)+\int_{0}^{T} \mathcal{L}(\phi(t \mid K), K, t) d t
$$

is minimised, where $\Gamma$ and $\mathcal{L}$ are obtained from the corresponding terms of (14) in an obvious manner.

Note that the dimension of the deterministic state $\phi$ is now $\left(n^{2}+3 n\right) / 2$. For convenience, let this deterministic optimal parameter selection problem (subject to the parameter constraint (3) and probabilistic state constraint (17)) be referred to as the problem (DP).

The problem (DP) is a special case of the general class of combined parameter selection and optimal control problems considered in [18]. Many interesting theoretical results are now available in the literature such as $[1,4]$ and $[5]$. The numerical solution to this class of problem can easily be obtained by the software package MISER [7, 9]. Essentially, after appropriate control parameterisation analysis, this class of problem can be reduced to the form of a purely optimal parameter selection problem for a 
dynamical system whose state $x$ is governed by

$$
\begin{aligned}
\frac{d x}{d t} & =f(x(t), K, t), \quad t \in[0, T] \\
x(0) & =x^{0}(K) .
\end{aligned}
$$

The cost functional $g_{0}$ and the constraints $g_{i}, i=1, \ldots, N$ may be expressed in canonical form as

$$
g_{i}(K)=\Gamma_{i}(x(T), K)+\int_{0}^{T} \mathcal{L}_{i}[x(t), K, t] d t, i=0,1,2, \ldots, N .
$$

The problem thus seeks to select the parameter $K$ such that $g_{0}$ is minimised subject to the constraints $g_{i}(K)=0, i=1, \ldots, N_{e}, g_{i}(K) \geqslant 0, i=N_{e}+1, \ldots, N_{h}$. It is easy to see that the problem $D P$ as defined by (19), (3) and (17) is a special case of this canonical formulation. In principle, the problem can be regarded as a constrained nonlinear programming problem. In practice, however, due to the non-explicit dependence of $g_{i}, i=0,1, \ldots, N$ on the parameter $K$, the gradients of these functionals have to be computed in a roundabout way. The details of these derivations can be found in [7]. We shall summarise the results in the following steps:

For each iteration point $K$, and for each $g_{i}, i=1, \ldots, N$ :

STEP 1 . Solve the differential equation (20) forward from 0 to $T$.

STEP 2. Evaluate the corresponding costate $\lambda_{i}$ by integrating the costate equation defined by

$$
\begin{aligned}
\dot{\lambda}_{i}^{T} & =-\frac{\partial \mathcal{L}_{i}}{\partial x}-\lambda_{i}^{T} \frac{\partial f}{\partial x} \\
\lambda_{i}^{T}(T) & =\frac{\partial \Gamma_{i}}{\partial x(T)}
\end{aligned}
$$

backward from $T$ to 0 .

STEP 3. Compute the gradient of $g_{i}$ by

$$
\nabla_{K} g_{i}=\frac{\partial \Gamma_{i}}{\partial K}+\frac{\partial x^{0}}{\partial K} \lambda_{i}(0)+\int_{0}^{T}\left[\frac{\partial \mathcal{L}_{i}}{\partial K}+\lambda_{i}^{T} \frac{\partial f}{\partial K}\right] d t .
$$

With this gradient information, the optimal parameter selection problem may thus be solved using any standard algorithm for constrained nonlinear programming problems. An efficient code used here is based on the sequential quadratic programming algorithm [15].

To finish off this section, we wish to note that similar results are also valid for the problem (P) with the assumption that $\xi^{0}$ is a deterministic vector rather than a Gaussian distributed random vector. In this situation, the initial conditions for (10) and (11) are, respectively, replaced by $\xi^{0}$ and 0 . 


\section{Numerical EXamples}

For illustrative purposes, we consider a second order system with white noise input $n(t)$ of constant spectral density:

$$
\frac{d^{2} \xi(t)}{d t^{2}}+2 \zeta \omega \frac{d \xi(t)}{d t}+\omega^{2} \xi(t)=u(t)+n(t)
$$

with initial conditions:

$$
\xi(0)=1, \frac{d \xi(0)}{d t}=0
$$

where

$$
E\{n(t) n(\tau)\}=\theta \delta(t-\tau)
$$

Here $\theta$ is a positive constant, and $\delta(t)$ is the delta function.

The design problem is to select a constant state feedback control law of the form:

$$
u(t)=K_{1} \xi(t)+K_{2} \frac{d \xi(t)}{d t}
$$

such that the expected value of a quadratic cost functional $J(K)$ is minimised over $m$ natural undamped period, where

$$
J(K)=E\left\{\int_{0}^{2 m \pi / \omega}\left\{\frac{1}{2} \omega^{2}(\xi(t))^{2}+\frac{1}{2}\left(\frac{d \xi(t)}{d t}\right)^{2}+\frac{1}{2} R(u(t))^{2}\right\} d t\right\} .
$$

We stress the importance of the constant gain as well as finite $m$ here because if $K_{i}$ are allowed to vary with time (with finite $m$ ), or if $m$ is infinite (with $K_{i}$ constant), the problem is amenable to the standard linear quadratic theory. To proceed, we may rescale the time with respect to $1 / \omega$ and express the scalar system in state space form:

where

$$
\begin{gathered}
d \xi(t)=A \xi(t) d t+B u(t) d t+D d w(t) \\
\xi(0)=\xi^{0}=\left[\begin{array}{l}
1 \\
0
\end{array}\right] \\
A=\left[\begin{array}{cc}
0 & 1 \\
-1 & -2 \zeta
\end{array}\right], B=\left[\begin{array}{l}
0 \\
1
\end{array}\right], D=\left[\begin{array}{l}
0 \\
1
\end{array}\right], K^{T}=\left[K_{1}, K_{2}\right]
\end{gathered}
$$

$u(t)=K^{T} \xi(t)$, and $w(t)$ is the Wiener process with

$$
E\left\{(w(t))^{T} w(\tau)\right\}=\int_{0}^{\min (t, \tau)} \theta d s
$$


Thus the closed loop system matrix is

$$
\tilde{A}(k)=A+B K=\left[\begin{array}{cc}
0 & 1 \\
K_{1}-1 & K_{2}-2 \zeta
\end{array}\right]
$$

and the nondimensionalised cost functional is

$$
\begin{aligned}
J(K)= & E\left\{\int_{0}^{2 m x} \frac{1}{2}(\xi(t))^{T}\left[I+R K K^{T}\right] \xi(t) d t\right\} \\
= & \frac{1}{2} \int_{0}^{2 m \pi}\left\{\left(\psi_{11}(t \mid K)+\left(\mu_{1}(t \mid K)\right)^{2}\right)\left(1+R\left(K_{1}\right)^{2}\right)\right. \\
& +2 R K_{1} K_{2}\left[\psi_{12}(t \mid K)+\mu_{1}(t \mid K) \mu_{2}(t \mid K)\right] \\
& \left.+\left[\psi_{22}(t \mid K)+\left(\mu_{2}(t \mid K)\right)^{2}\right]\left(1+R\left(K_{2}\right)^{2}\right)\right\} d t,
\end{aligned}
$$

where the state $\mu=\left[\begin{array}{l}\mu_{1} \\ \mu_{2}\end{array}\right]$ and $\psi=\left[\begin{array}{ll}\psi_{11} & \psi_{12} \\ \psi_{12} & \psi_{22}\end{array}\right]$ are governed by the differential equations:

$$
\begin{gathered}
\frac{d \mu(t)}{d t}=\bar{A}(k) \mu(t) \\
\mu(0)=\xi^{0}=\left[\begin{array}{l}
1 \\
0
\end{array}\right]
\end{gathered}
$$

and

$$
\begin{aligned}
\frac{d \psi_{11}(t)}{d t} & =2 \psi_{12}(t) \\
\frac{d \psi_{12}(t)}{d t} & =\psi_{22}(t)+\psi_{11}(t)\left(K_{1}-1\right)+\psi_{12}(t)\left(K_{2}-2 \zeta\right) \\
\frac{d \psi_{22}(t)}{d t} & =2 \psi_{12}(t)\left(K_{1}-1\right)+2 \psi_{22}(t)\left(K_{2}-2 \zeta\right)+\theta \\
\psi_{11}(0) & =0, \psi_{12}(0)=0, \psi_{22}(0)=0
\end{aligned}
$$

A quick implementation of MISER on this problem with $\zeta=0.1, R=2$, and $2 m \pi=10$ furnishes the following solutions for different values of $\theta$. 


\begin{tabular}{|c|c|c|}
\hline$\underline{\theta}$ & $-K_{1}^{*}$ & $-K_{2}^{*}$ \\
\hline 0.0 & 0.2279 & 0.7969 \\
\hline 0.1 & 0.1989 & 0.7379 \\
\hline 0.2 & 0.1864 & 0.7207 \\
\hline 0.3 & 0.182 & 0.7190 \\
\hline 0.4 & 0.181 & 0.718 \\
\hline 0.5 & 0.18 & 0.713 \\
\hline 1.0 & 0.18 & 0.708 \\
\hline 2.0 & 0.18 & 0.703 \\
\hline 5.0 & 0.18 & 0.700 \\
\hline
\end{tabular}

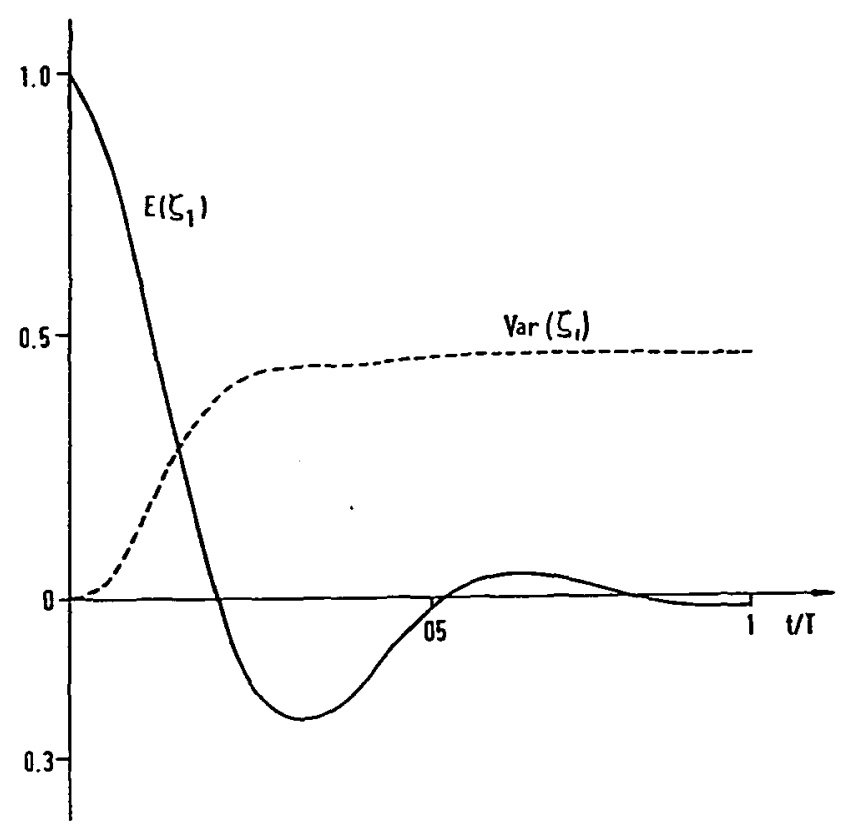

Figure 1

The computed solutions are found to be insensitive to the magnitude of $\theta$ when $\theta$ is greater than 0.5. Consider the case when $\theta=0$ (that is without noise) and $m=\infty$. By applying the usual linear quadratic theory, the steady state gain can easily be computed to be $K_{1}^{*}=-0.2247$ and $K_{2}^{*}=-0.7947$. This steady state gain vector is very close to the solution of the original problem (that is $T=2 m \pi<\infty$ ) with 
$\theta=0$ obtained from the proposed method. As $\theta$ increases, the gain reduces gradually to an asymptotic value as shown above. The optimal mean state for $\xi_{1}(t)$, (that is, $\left.E\left(\xi_{1}^{*}(t)\right)=\mu_{1}^{*}(t)\right)$, and optimal variance for $\xi_{1}(t)$, (that is $\operatorname{Var}\left(\xi_{1}^{*}(t)\right)=\psi_{11}^{*}(t)$ ), as functions of $t$ are plotted in Figure 1 for the case of $\theta=1$.

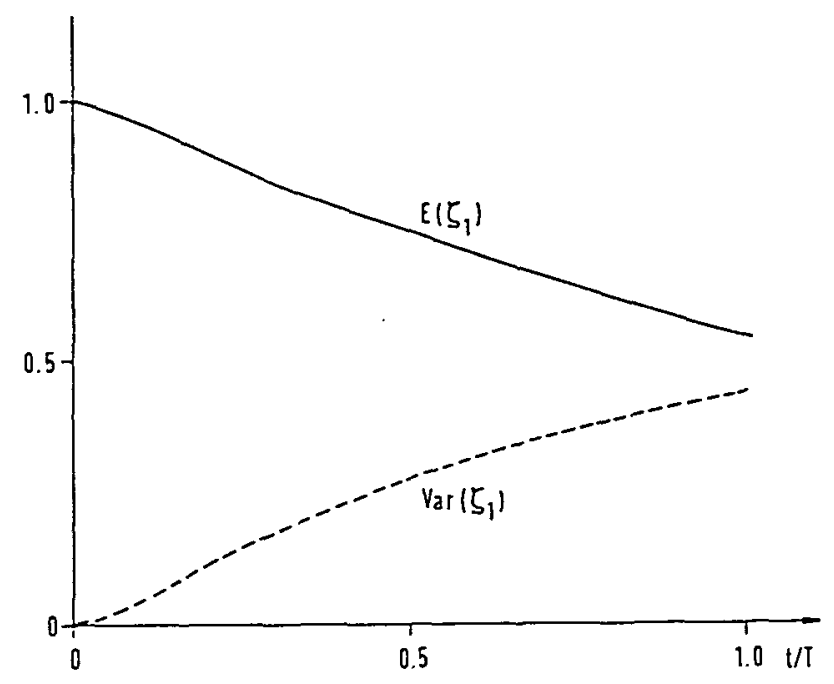

Figure 2

To go a step further, we impose the following probabilistic state constraint:

$$
\operatorname{Prob}\left\{\xi_{1}(t) \geqslant \varepsilon\right\} \geqslant \triangle,
$$

which, after appropriate transformation, reduces to

$$
\operatorname{erf}\left[\frac{\mu_{1}(t \mid K)-\varepsilon}{\left(2 \psi_{11}(t \mid K)\right)^{1 / 2}}\right] \geqslant 2 \Delta-1
$$

for all $t \in[0,2 m \pi]$.

If $\varepsilon=0$ and $\Delta=0.8$, the optimal solution for $R=2$ and $\theta=1$ is $K^{*}=\left[K_{1}^{*}, K_{2}^{*}\right]^{T}$ with $K_{1}^{*}=0.7813$ and $K_{2}^{*}=-3.407$.

The optimal mean state and variance for $\xi_{1}(t)$ are plotted in Figure 2. The drastic change in solution structure from that of Figure 1 is noted. A quick check on the solution indicates that the probabilistic state constraint is active only at the end point where $E\left(\xi_{1}\right)$ is minimal.

To account for a random initial condition for $\xi^{0}$, we compute the constrained example again, using a non-zero initial condition of $M^{0}=0.2 I$. Optimum values for 


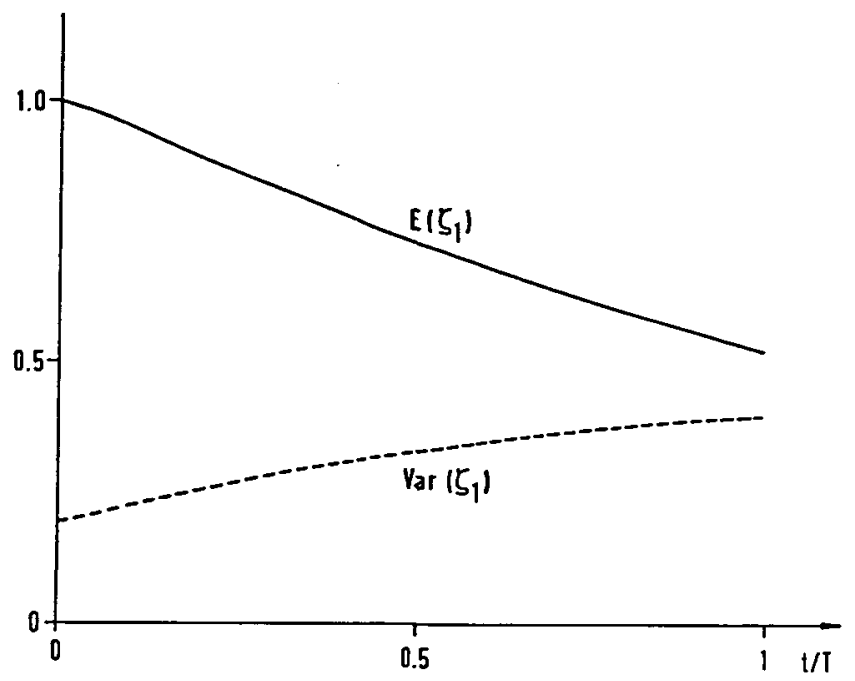

Figure 3

the parameter change slightly to $K_{1}^{*}=0.7426$ and $K_{2}^{*}=-3.8264$. The optimum mean state and variance for $\xi_{1}(t)$ are plotted in Figure 3. Despite the non-zero initial condition, the variance of $\xi_{1}(t)$ grows to about the same as the previous case of zero initial condition. This is to be expected since the probability state constraint is only active at the terminal time.

\section{REFERENCES}

[1] N.U. Ahmed, Elements of Finite-dimensional Systems and Control Theory (Longman Scientific \& Technical, Harlow, 1988).

[2] N.U. Ahmed and N.D. Georgenas, 'On optimal parameter selection', IEEE Trans. Automat. Control AC-18 (1973), 313-314.

[3] B.D.D. Anderson and J.B. Moore, Linear Optimal Control (Prentice-Hall, Englewood Cliffs, 1971).

[4] L. Cesari, Optimization Theory and Applications (Springer-Verlag, Berlin, Heidelberg, New York, 1983).

[5] B.D. Craven, Mathematical Programming and Control Theory (Chapman and Hall, London, 1978).

[6] J. Dolezal, 'On the solution of optimal control problems involving parameters and general boundary conditions', Kybernetika 17 (1981), 71-81.

[7] C.J. Goh and K.L. Teo, MISER: An Optimal Control Software (Applied Rsearch Corporation, National University of Singapore, 1987).

[8] C.J. Goh and K.L. Teo, 'Control parametrization: A unified approach to optimal control problems with general constraints', Automatica 24 (1988), 3-18. 
[9] C.J. Goh and K.L. Teo, 'MISER: A Fortran program for solving optimal control problems', Adv. Engrg. Software 10 (1988), 90-98.

[10] L.S. Jennings and K.L. Teo, 'A computational algorithm for functional inequality constrained optimization problems', Automatica (1990) (to appear).

[11] H. Kwakernaak and R. Sivan, Linear Optimal Control System (Wiley-Interscience, New York, 1972).

[12] , 'Linear-Quadratic-Gaussian Problems', Special Issue, IEEE Trans. Automat. Control AC-16 (1971).

[13] D.W. Reid and K.L. Teo, 'Optimal parameter selection of parabolic systems', Math. Oper. Res. 5 (1980), 467-474.

[14] D.L. Russell, Mathematics of Finite-Dimensional Control Systems (Marcel Dekker, New York, 1979).

[15] K. Schittkowski, 'NLPQL: A Fortran subroutine for solving constrained nonlinear programming problems', Oper. Res. Ann. 5 (1985), 485-500.

[16] K.L. Teo and N.U. Ahmed, 'Optimal feedback control for a class of stochastic systems', Internat. J. Systems Sci. 5 (1974), 357-365.

[17] K.L. Teo and C.J. Goh, 'A simple computational procedure for optimization problems with functional inequality constraints', IEEE Trans. Automat. Control. AC-32 (1987), 940-941.

[18] K.L. Teo and C.J. Goh, 'A computational method for combined optimal parameter selection and optimal control problems with general constraints', J. Austral. Math. Soc. Ser. B 30 (1989), 350-364.

[19] L.J. Van Mellaert and P. Dorato, 'Numerical solution of an optimal control problem with a probability criterion', IEEE Trans. Automat. Control AC-17 (1972), 543-546.

\footnotetext{
Department of Mathematics

University of Western Australia

Nedlands, WA 6009

Australia
} 